\title{
Learning Based Spectrum Hole Detection for Cognitive Radio Communication
}

\author{
Zhengjia Xu \\ School of Aerospace, \\ Transport \& Manufacturing \\ Cranfield University \\ MK43 0AL, UK \\ Zhengjia.Xu@cranfield.ac.uk
}

\author{
Ivan Petrunin \\ School of Aerospace, \\ Transport \& Manufacturing \\ Cranfield University \\ MK43 0AL, UK \\ i.petrunin@cranfield.ac.uk
}

\author{
Antonios Tsourdos \\ School of Aerospace, \\ Transport \& Manufacturing \\ Cranfield University \\ MK43 0AL, UK \\ a.tsourdos@cranfield.ac.uk
}

\author{
Shahid Ayub \\ School of Aerospace, \\ Transport \& Manufacturing \\ Cranfield University \\ MK43 0AL, UK \\ Shahid.Ayub@cranfield.ac.uk
}

\begin{abstract}
This paper proposes a novel learning based (LB) solution for detection and quantification of spectrum holes in periodic communications of unmanned aerial vehicles (UAVs). Instead of hypothesis testing after implementation of spectrum sensing methods, the implemented LB solution based on spectral correlation function (SCF) uses region convolutional neural network (R-CNN) for extracting quantitative parameters of the spectrum holes.

The proposed LB approach is implemented using GoogLeNet architecture for the wide band detection in the scenario of orthogonal frequency division multiplexing (OFDM) communication system with the additive white Gaussian noise (AWGN) channel model. The simulation of single input single output (SISO) communication system with spectrum holes is presented. Examples of wide band detection results for both SISO and multiple input multiple output (MIMO) systems are shown and the proposed LB detector is found to be fairly accurate in identification of spectrum holes. By analyzing the training performance, the GoogLeNet architecture, along with its hyperparameter configurations and training dataset is validated. We also demonstrated that our LB detector is resilient to the AWGN environment by analyzing the precision and recall curves, average precision and mean relative error (MRE) versus signal noise ratio (SNR).
\end{abstract}

Index Terms-learning based sensing, cognitive radio, R-CNN, cyclostationary feature sensing, UAV communication

\section{INTRODUCTION}

With a growing demand of UAV applications, an agile and intelligent communication for UAS becomes necessary in order to meet the increasing requirements and address the problem of limited spectrum resources. Cognitive radio (CR) proposed in [1] is regarded as a potential solution for achieving the maximization of spectrum usage and improving the communication efficiency, which allows secondary users (SUs) opportunistically access the spectrum when primary users (PUs) are vacant. Spectrum holes indicate the availability of spectrum resource in terms of time and frequency domain. One principle in the CR concept is that the SUs are not permitted to interfere PUs' communications. The activity of spectrum access can be easily implemented with the assumption of having periodic inherent in spectrum holes. Therefore, we assume in this paper that the spectrum holes have periodic behaviours.
One of the main functions of the cognitive communication system is to detect the presence of spectrum holes in the radio frequency (RF) environment. Spectral awareness is an important step to gain access to spectrum and it is classified into passive (rely on external services, such as radio environment map (REM) [2]) and active awareness that is commonly known as spectrum sensing [3]. The typical sensing methods are energy detection, matched filtering detection, wide-band sensing, robust sensing, cyclostationary feature sensing, compressed sensing, etc. Complete reviews of spectrum sensing technology are explained in [4] [5].

As one of the most popular detection methods, cyclostationary feature detection is commonly utilized for spectrum sensing of communication signals by demonstrating the inherent periodic behaviour with the following formulation of a hypothesis and decision making based on comparison with a threshold [6] [7].

Selection of threshold especially a dynamic threshold in the detector to determine the existence of potential signal is one of the main challenges for the conventional cyclostationary feature detector [8] [9] [10]. Assumptions on an accurate channel model when designing the detector are also necessary, which are difficult to satisfy in practice. Therefore, an intelligent detector with its adaptive threshold adjustment to the environment is an important part of cognitive communication system.

Another challenge of spectrum hole detection is in the need of its implementation for wide band sensing scenario. Most cyclostationary based sensing technologies assume single channel or in a narrow band. There are some methods that expand the bandwidth of cyclostationary detector, e.g. by monitoring multiple channels [11] or using channel scanning strategy [12]. However, above solutions are computationally expensive when the signal frequency is unknown. Therefore, a more efficient strategy based on searching algorithms, e.g. employed in [13] [14] for object detection, is implemented in our scenario.

Object detection technique is frequently utilized for detecting pedestrian or vehicles in computer vision with two main objectives: classify objects and locate them in the image. The conventional approach of object detection implements a 


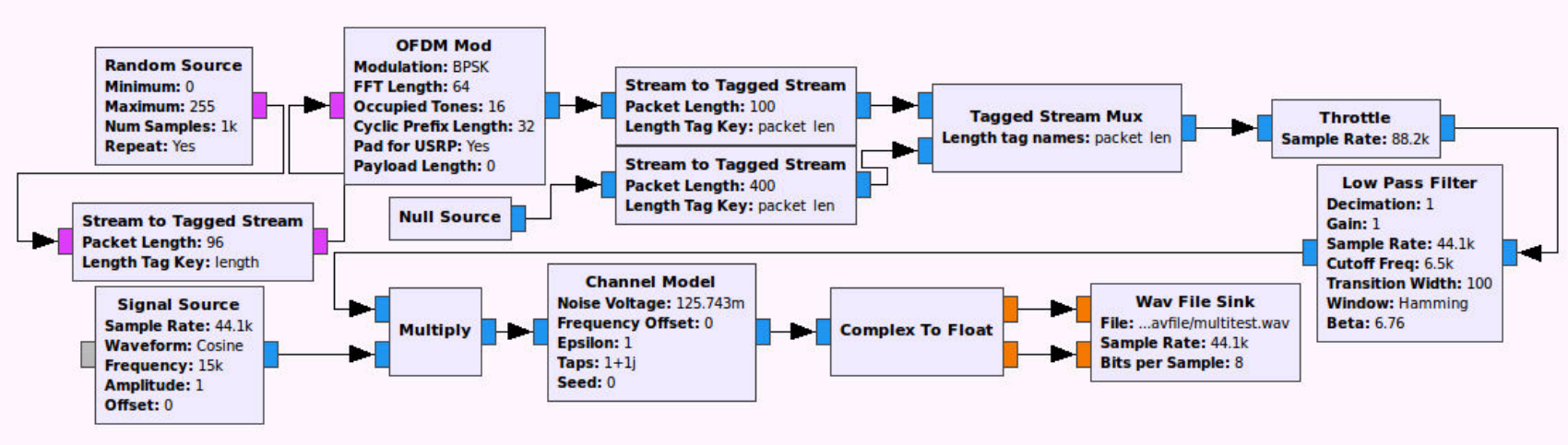

Fig. 1. GNU Radio diagram of simulated communication system (screenshot from GRC)

sliding window as the searching strategy [15] (similar with the channel scanning in [12]), and then applies some handcrafted techniques, such as scale invariant feature transform (SIFT) [16], histogram of oriented gradient (HOG) [17], etc., to extract feature maps manually. Such convolutional object detection methods are complex in computation and characterized by low precision. Therefore, a neural network based solution, i.e. region based convolutional neural network (R-CNN), is proposed in [18] to solve the above drawbacks and is regarded as one of the fundamental methods for object detection.

In this paper, we integrate R-CNN architecture with cyclostationary feature analysis to propose a learning based (LB) solution for detecting spectrum holes in the communication system and extracting their quantitative parameters. As a datadriven approach, the intelligent selection of threshold and the adaptive detector are embedded in the learning procedure, which does not require explicit knowledge of a theoretic channel model. With assistance of automatic decision making in deep learning, our solution is expected to be resilient to the environmental factors.

The remaining part of this paper is organized as follows. In Sec. II, we present the simulation of communication system and the spectrogram of the desired communication signal. In Sec. III, we present the SCF function together with its visualized result. The learning based solution, along with GoogLeNet and R-CNN, is illustrated in Sec. IV. The performance of LB detector is analyzed in Sec. V and the conclusions are presented in Sec. VI.

\section{Simulation of COMmunication System With SPECTRUM HOLES}

In order to simulate a practical case of communication scenario having complex signal patterns, we utilize orthogonal frequency division multiplexing (OFDM) as the modulation scheme, due to its immunity to selective fading, resilience to inter-symbol interference (ISI) and higher throughput in comparison with single band communication methods [19].

Simulation of the communication system in this study is performed using an open-source software, GNU Radio [20]
- a powerful tool for simulating communication systems or building applications, e.g. for developing suitable signal processing programs and for simulation of PU transmitter and receiver in [20].

GNU Radio companion (GRC) is a graphic user interface (UI) of GNU Radio. By adding a series of signal processing blocks in accordance to the application requirements and creating so called flowgraph with example that can be seen in Fig. 1, development of communication system becomes significantly simpler, making thus GRC attractive for use for the development purposes in both academia and industry. Communication signal generated for training and testing in this paper is obtained with use of GRC as a platform.

The diagram of single input single output (SISO) communication system at the transmitter side is presented in Fig. 1. This system generates a desired data stream which interleaves spectrum holes with a fixed periodicity. Some main blocks of the framework in Fig. 1 are explained below.

The data for transmitting is randomly created with a 'random source' block to avoid a repeated pattern. Signal modulation is implemented by a 'OFDM Mod' block, where its important parameters, e.g. modulation type, FFT length, occupied tones, cyclic prefix (CP) length, payload length, are explained in [20]. We generate spectrum holes in the transmitted signal by interleaving blank data fragments and OFDM data fragments and use a 'Tagged Stream Mux' block to combine these two fragments together. Low pass filter (LPF) is designed for standardizing the bandwidth of signal. Another signal source with cosine waveform is utilized for shifting the signal to a desired frequency. We introduce the additive white Gaussian noise (AWGN) channel model into simulation by using a 'Channel Model' block. The finalized signal is saved through a 'wav file sink' block in I/Q data format.

Using the simulated system in Fig. 1 we have generated communication signal with the following parameters: signal to noise ratio (SNR) is $E b / E 0=15 d B$, number of tones of the OFDM signal is 14 , central frequency of signal is 15000 $\mathrm{Hz}$, bandwidth of signal is $10000 \mathrm{~Hz}$ and cyclic frequency of spectrum hole is $88 \mathrm{~Hz}$. The spectrogram of the simulated signal with above conditions is shown in Fig. 2. 


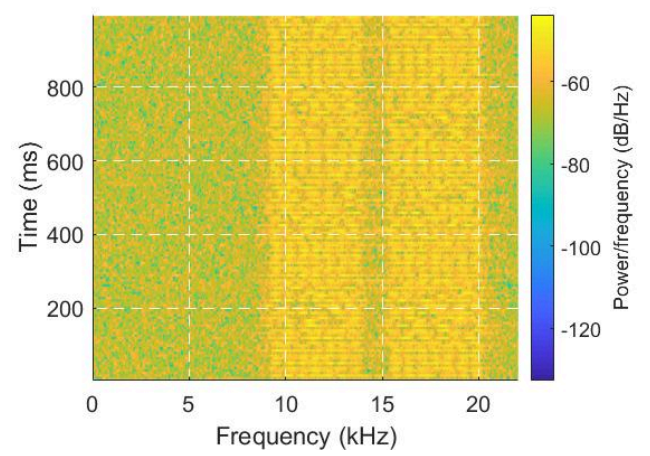

Fig. 2. Spectrogram of the generated OFDM signal for $E b / E 0=15 d B$

As is displayed in Fig. 2, the generated OFDM signal demonstrates its characteristics, such as 14 tones, central frequency of $15000 \mathrm{~Hz}$, bandwidth of $10000 \mathrm{~Hz}$, etc., that match the desired configuration. The periodic intervals during the transmission signals are observable, which proves the success of embedding spectrum holes using the simulation framework presented in Fig. 1.

\section{SPectral Correlation Function}

With the assumption of periodicity in spectrum holes, cyclostationary feature sensing is expected to outperform conventional methods, e.g. energy detection, especially in low SNR regions due to better noise immunity [21]. Spectral correlation function (SCF) is one of the most common methods to extract cyclic features in the frequency domain and is introduced in this section.

According to [22], the cyclic autocorrelation function (CAF) $R_{X}\left[\tau ; \alpha_{i}\right]$ of a discrete signal $X[n]$ is defined in the time domain as follows:

$$
R_{X}\left[\tau ; \alpha_{i}\right]=\lim _{L \rightarrow \infty} \frac{1}{L} \sum_{n=-L / 2}^{L / 2} R_{X}[n, \tau] e^{-j 2 \pi \alpha_{i} n \Delta}
$$

where $\alpha$ is cyclic frequency belonging to the cyclic frequency set $\mathcal{A} ; \tau$ is the time lag; $\Delta$ corresponds to the time interval; $L$ is the length of the signal.

SCF of $x[n]$ is the Fourier transform (FT) of CAF [23] with the expression as: $\Phi_{X}(f ; \alpha)=\mathcal{F}\left[R_{X}[\tau ; \alpha]\right]$. Practical method of estimating SCF is based on using an averaged periodogram, hence the estimated SCF is given as follows [24]:

$$
\begin{array}{r}
\hat{\Phi}_{X}(f ; \alpha)=\frac{1}{K \Delta} \sum_{k=0}^{K-1} \mathcal{X}_{n_{w}}^{k}[f+(1-\beta) \alpha] . \\
\mathcal{X}_{n_{w}}^{k}[f-\beta \alpha]^{*}
\end{array}
$$

where $\mathcal{X}_{n_{w}}^{k}$ is obtained from short time Fourier transform (STFT) of $X[n]$ with an averaging window defined by coefficients $k_{t h} ; K=I\left[\left(L-n_{w}\right) / R\right]+1$ with $L$ is signal length, $n_{w}$ is window width, and $R$ is a sample index allowing overlaps. $I$ is a ceil function that returns the smallest integer value that is bigger or equal to the input value.
The three dimensional representation of the SCF of the simulated communication signal (generated using the system in Fig. 1) is shown in Fig. 3.

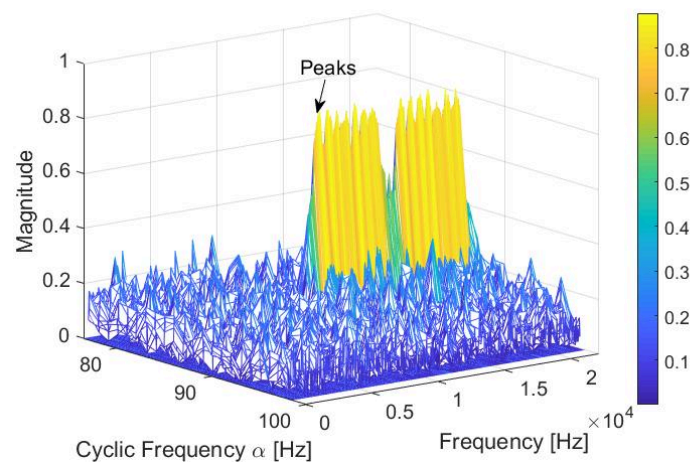

Fig. 3. Spectral correlation function of the generated OFDM signal

As one can see from the Fig. 3, the CAF of the desired communication signal has an observable pattern in a form of multiple peaks. We can easily extract some other characteristics, e.g. the central frequency is around $15000 \mathrm{~Hz}$, bandwidth is $10000 \mathrm{~Hz}$, number of tone (presented as peaks) is 14, and cyclic frequency is $88 \mathrm{~Hz}$. The above results are in accordance to our desired configuration and this validates the effectiveness of SCF method in extraction of cyclic patterns of the simulated signal.

\section{Learning BASEd Detection Solution}

In this paper, we propose to use a learning based scheme to detect and extract spectrum holes, where R-CNN is considered as a intelligent detector for spectrum sensing because of its efficiency in 'recognition using regions' [18]. The framework of the proposed LB solution is presented in Fig. 4.

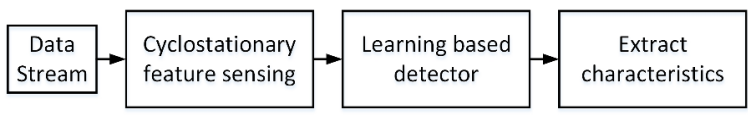

Fig. 4. The framework of R-CNN based detection

As illustrated in Fig. 4, the framework of the proposed LB solution contains the following parts: data stream generator (presented in Sec. II), the cyclostationary detector (SCF based sensing explained in Sec. III), a learning based detector (R$\mathrm{CNN}$ is explained in Sec. IV-B) and the extractor of spectrum hole parameters (explained below).

The first step of our LB solution is to use the SCF, defined by equation (2), to extract the feature map of the processed signal. At the second step, by performing the deep learning based detection, the presence of spectrum hole is detected and its characteristics are extracted.

The representation of R-CNN output (called bounding box) is commonly defined as rectangle described by parameters $(t, l, w, h)$, where $t$ and $l$ are the top and left corner indices, and $w$ and $h$ are the width and height of the rectangle, respectively. 
Spectrum hole is defined in terms of its parameters: cyclic frequency $\alpha$, frequency $f$ and bandwidth $R$. The extraction of the above parameters follows the equations of $\alpha=$ $f_{m x}\{t+w / 2\}, f_{c}=f_{m y}\{l+h / 2\}$, and $R_{a}=f_{m y}\{w\}$, where $f_{m x}$ and $f_{m y}$ are the mapping functions in the horizontal and vertical directions.

\section{A. GoogLeNet Architecture}

Since GoogLeNet has been proposed in [25], its concept of designing dense components to cover the optimal sparse structure increases the detection accuracy and helped it to win the competition in the ImageNet Large-Scale Visual Recognition Challenge 2014 (ILSVRC14) [25] and stimulated its application in different classification and detection scenarios, e.g. for lung cancer detection [26], illegal building detection [27], etc. The state-of-art of GoogLeNet is attributed to its implementation of inception layer, which makes the GoogLeNet capable of improving performance while with a more concise architecture. Therefore, this paper implements GoogLeNet as the deep neural network for detecting spectrum holes.

The complete GoogLeNet architecture is shown in Fig. 5; details of its configuration are discussed in [25].

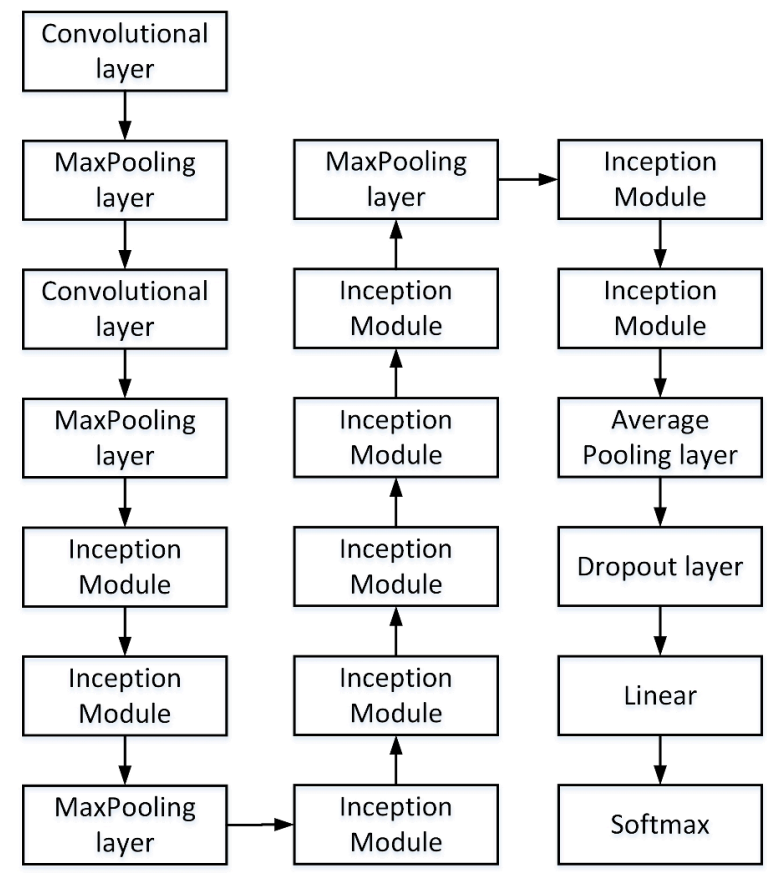

Fig. 5. GoogLeNet architecture

From the architecture perspective, the biggest difference between GoogLeNet and other popular networks, e.g. AlexNet [28], is the novel design of inception module. The inception module can increase the complexity of network while decrease the number of parameters at the same time. The concept of inception module is using "readily available dense component", i.e. the combination of convolutional layers and pooling layers, to approximate and find the optimal local sparse construction.

According to [25], two versions of inception module are implemented, that are a naive version and an inception module with dimensionality reduction. The necessity of designing of the second inception module is related to the fact that the naive version would introduce the increasing number of output from stage to stage, leading to the computation explosion within a few stages. The reduction of dimension is achieved by adding an extra $1 * 1$ convolutional layer before each existing convolutional layer. Such $1 * 1$ convolutional layer can not only be used for up-sampling or down-sampling the feature map, but also for introducing closer combinations of feature maps and increase nonlinearity of the characteristic. Architectures of these two versions are explained in Section 4 of work [25].

\section{B. Region Based Convolutional Neural Network}

The reason $\mathrm{R}-\mathrm{CNN}$ is adopted in this study is because of the 'recognition using regions scheme that R-CNN effectively implements in [18]. Such scheme is based on searching algorithms, e.g. selective search [13] or edge box based search [14], that generate numerous region proposals, also called region of interests (RoIs), with rectangular boxes as labels. It is known that naive approaches, such as exhaustive search over the whole instance, would be expensive, in particular for large-scale dimensions. By comparing with other common searching algorithms in terms, for example, of detection rate versus number of proposals and desired intersection over union (IoU) thresholds, edge box based searching method is found to be faster and more accurate than others, e.g. selective search or randomized Prims and Rahtu [18]. Therefore, we implement edge box based searching algorithm here to generate region proposals.

A framework of the implemented R-CNN approach is presented in Fig. 6.

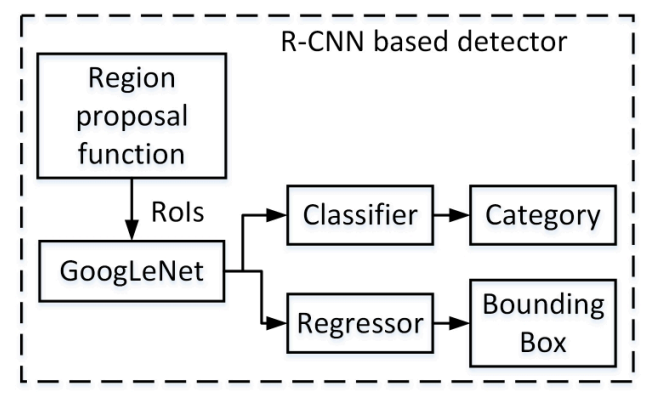

Fig. 6. Framework of R-CNN for image detection

As illustrated in Fig. 6, after generating RoIs by the region proposal function (edge box based searching), a deep neural network (GoogLeNet) is implemented here to extract feature maps for each RoI. After that we perform the classification and regression operations on each RoI in order to detect existence of spectrum hole and evaluate location of the spectrum hole in two dimensional space (i.e. over frequency and cyclic frequency). The output of R-CNN, containing category and the label with position, is presented by a bounding box of a rectangular shape. The spectrum hole characteristics are then extracted from this bounding box. 


\section{Example of R-CNN Based Detection}

Results of the R-CNN implementation for spectrum holes detection, marked by yellow rectangle in the spectrogram are shown in Fig. 7 for SISO system and AWGN environment with $E b / N 0=0 d B$. The corresponding R-CNN based detection for MIMO system is shown in Fig. 8.

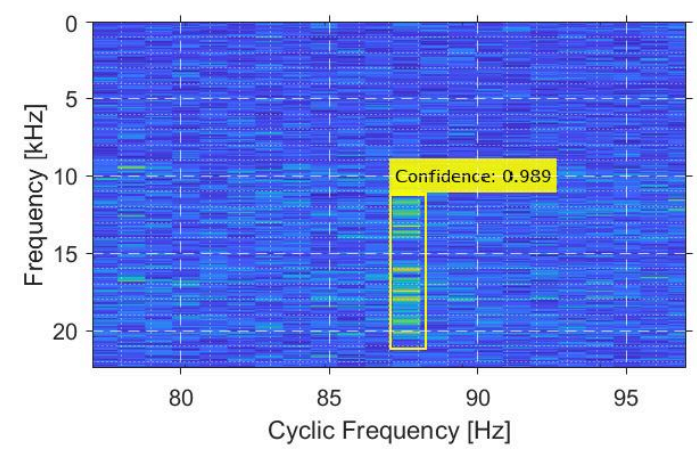

Fig. 7. Example of R-CNN based detection for SISO communication system at $E b / N 0=0 d B$

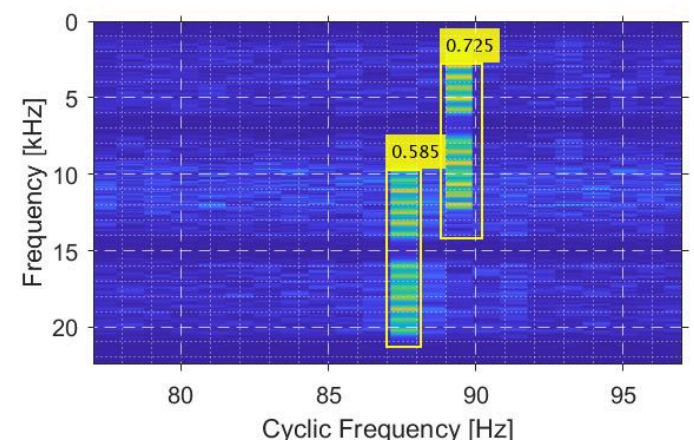

Fig. 8. Example of R-CNN based detection for MIMO communication system at $E b / N 0=15 d B$

As it can be seen from Fig. 7, the yellow rectangular box, i.e. bounding box, covers most of the SCF pattern. The quantified parameters, i.e. cyclic frequency $\alpha$, central frequency $f_{c}$ and bandwidth $R_{a}$, can be extracted from the figure with values of $88 \mathrm{~Hz}, 16200 \mathrm{~Hz}$ and $9900 \mathrm{~Hz}$, respectively. Comparing with our desired values, i.e. $88 \mathrm{~Hz}, 15000 \mathrm{~Hz}$ and $10000 \mathrm{~Hz}$, the relative errors of $\alpha, f_{c}$ and $R_{a}$ are $0 \%, 9.45 \%$ and $1 \%$. The low error values demonstrate the possibility of successful identification of SCF pattern in wide bandwidth, and also prove the effectiveness of R-CNN based approach for the spectrum holes detection. High confidence score of 0.989 demonstrates the degree at that the detection result can be trusted. Details of calculating the confidence score are explained in the [29].

In order to simulate a MIMO communication system, we generate another OFDM signal by adding a new 'random source' block together with its peripherals and its blank data fragments in the framework Fig. 1 with the configurations of $E b / N 0=15 d B, \alpha=90 \mathrm{~Hz}, f_{c}=7000 \mathrm{~Hz}$ and
$R_{a}=1000 \mathrm{~Hz}$, the spectrogram for MIMO system with its detection result is illustrated in the Fig. 8. We can conclude from this figure that our LB detector is also effective for MIMO communication system in contrast to convolutional cyclostationary detectors that will struggle in such cases.

\section{Performance Analysis}

Due to the fact that our LB solution requires an additional training phase, the performance analysis of our LB detector is carried out from both training and detection perspectives.

\section{A. Training Performance}

The examination of the LB detector in training phase can be useful to verify the performance of neural network architecture, hyper-parameter (whose value is set before the training phase [30]) configuration and the integrity of dataset. Hyper-parameter configuration is set as follows: minimum batch size is 20 , initial learning rate is 0.0001 , maximum epoch size is 10 , negative overlapping range is $[0.1,0.3]$, and positive overlapping range is $[0.5,1]$.

For analysis of the behaviour of the implemented neural network architecture (GoogLeNet) after each iteration of optimization, we employ here the loss function (described in [18]) and the accuracy curve that represents number of successful detections for the entire training dataset, when IoU of bounding box and ground truth is over 0.5 . The training performance result in terms of loss function is presented in Fig. 9 and the accuracy function result is presented in Fig. 10.

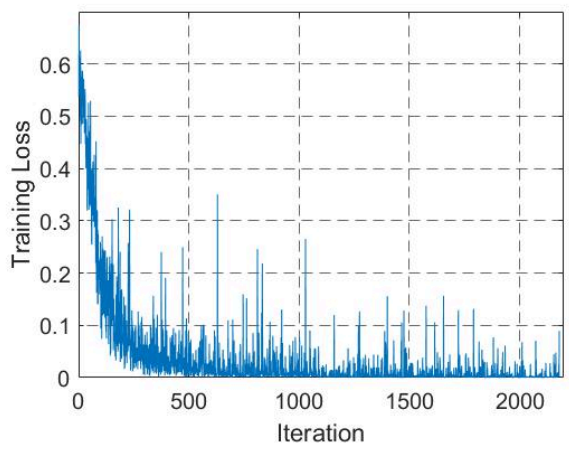

Fig. 9. Loss curve of training phase

For the loss curve displayed in Fig. 9 we can note that the loss curve declines at a fast rate in the first 250 iterations and then drops gently after 500 iterations. The decline rate of loss curve is becoming slower with increase in number of iteration. The final loss curve after 2000 iterations converges to a low value (around 0.001), which reveals the effectiveness of using GoogLeNet for the training along with the utilized hyperparameter configurations and the generated training dataset. Similar conclusions, e.g. the effectiveness of GoogLeNet, hyper-parameter configurations and dataset integrity, can be demonstrated by analyzing the accuracy curve in Fig. 10 as well. 


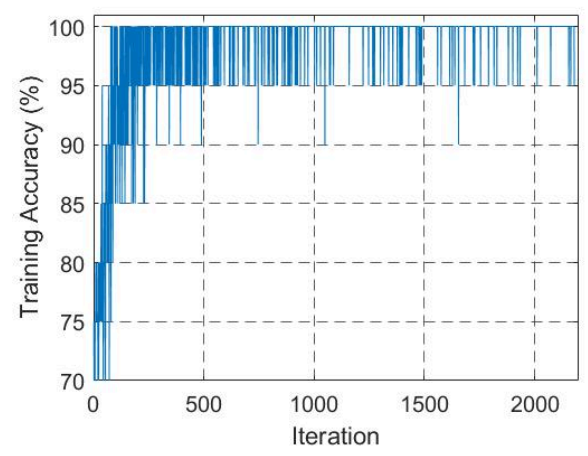

Fig. 10. Accuracy curve of training phase

It should be mentioned that some fluctuations displayed in the above curves are caused by the dropout function (shown in Fig. 5). The poor precision (i.e. 5\% in the Fig. 10) is a consequence of low value of one of the hyper-parameter, the mini-batch size, set to 20 .

\section{B. Detection Performance}

The performance of LB detector is examined by implementing the Precision-Recall (PR) curve, where the precision describes the retrieval ratio among relevant instances and recall describes the retrieval ratio among the full set of instances [31] [32]. Precision and recall are incompatible in most cases, thus the goal of improving the PR performance is expected to have the curve moving towards the upper-right-hand corner [33]. The PR results with AWGN channel model are presented in Fig. 11.

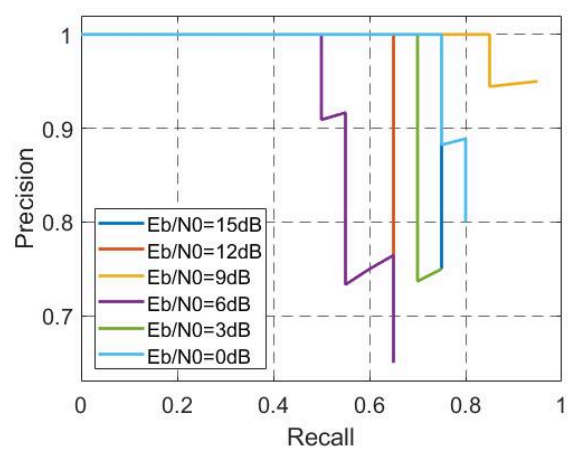

Fig. 11. Precision and recall curve of R-CNN based detector

As can be seen in Fig. 11, all the PR curves are in the upper-right-hand corner and cover a wide PR space (the area below the curve) under different $E b / N 0$ environments, which demonstrates a high probability of detection and resilience of our LB detector against AWGN environment. It is noticeable that the condition of $E b / N 0=6 d B$ demonstrates the poorest result here. In contrast to conventional cyclostationary detectors, e.g. SCF based detection in [34], our data-driven LB detector does not show any visible correlation between the performance of detector and SNR $(E b / N 0)$ value in this experiment.
In order to obtain the PR curve, we utilize average precision (AP) as the measure due to its sensitivity to the retrieval results [32]. The implementation of AP is achieved by calculating the PR space. Mean relative error (MRE) of the quantitative parameters, i.e. cyclic frequency $\alpha$, central frequency $f_{c}$ and bandwidth $R_{a}$, are also calculated with the results displayed in Table. I.

TABLE I

MRE AND AP RESULT WITH AWGN CHANNEL MODEL

\begin{tabular}{lllllll}
\hline $\mathrm{Eb} / \mathrm{N} 0(\mathrm{~dB})$ & 0 & 3 & 6 & 9 & 12 & 15 \\
\hline$\alpha(\%)$ & 1.09 & 1.41 & 2.17 & 1.66 & 1.65 & 1.89 \\
$f_{c}(\%)$ & 7.01 & 3.10 & 3.20 & 4.06 & 4.27 & 5.55 \\
$R_{a}(\%)$ & 7.88 & 9.10 & 9.25 & 4.53 & 7.22 & 8.25 \\
$\mathrm{AP}(\%)$ & 79.44 & 73.75 & 62.16 & 94.49 & 65.00 & 75.00 \\
\hline
\end{tabular}

By observing the AP results in Table. I, the smallest value of AP is $62.16 \%$ which corresponds to $E b / N 0=6 d B$. Meanwhile, comparing with the mean average precision (mAP) result, i.e. average of AP for each class, for image detection in [35] (around 71\% with VOC 2007 daset), our mAP (around $75 \%$ ) is over the value in [35]. Therefore, we can conclude that LB detector has a high performance from the AP perspective.

Since the worst performance is found for the condition of $E b / N 0=6 d B$ in Fig. 11, it is noticeable that the MRE results for parameters of $\alpha$ and $R_{a}$ (with values of $2.17 \%$ and $9.25 \%$ respectively in Table. I) are relatively high in comparison with values for other $E b / N 0$ values. Considering the bandwidth of cyclic frequency range of $21 \mathrm{~Hz}$ and the sensing bandwidth of $25000 \mathrm{~Hz}$, the maximum parameter errors of $\alpha, f_{a}$ and $R_{a}$ are $2.17 \%, 7.01 \%$ and $9.28 \%$ respectively in accordance to the frequency values of $0.4557 \mathrm{~Hz}, 1752.5 \mathrm{~Hz}$ and $2320 \mathrm{~Hz}$. Therefore, our LB detector is more sensitive to $\alpha$ because of its low MRE and less sensitive to $R_{a}$ because of its high MRE in this case. However, such sensitivity is relative to the bandwidth of cyclic frequency range and the sensing bandwidth.

The improvement of LB detector can be achieved using fine-tuning, e.g. by adding deep layer, optimizing kernel size and number of kernels, selecting appropriate initialization algorithms, etc. [36].

After reviewing the results we can note that there is no clear evidence that AWGN model has influence on the detector based on both PR and MRE results we have obtained. This may need to be further confirmed using larger testing dataset.

\section{CONCLUSION}

In this paper, we present a learning based sensing method for UAV communication system with capabilities of wide-band blind spectrum hole detection and immunity to white Gaussian noise environment. This method integrates conventional cyclostationay sensing technology with a R-CNN architecture to form an intelligent detector, where GoogLeNet is utilized as the convolutional neural network.

The proposed approach not only detects the existence of spectrum holes in wide band but also extracts their parameters: cyclic frequency, central frequency and bandwidth, for both 
SISO and MIMO communication systems. The simulation of a SISO communication system with OFDM signal interleaved with spectrum holes is performed and a common cyclostationary detection method (spectral correlation function) implemented here for feature extraction is found to be effective in such an analysis. The performance analysis of R-CNN based detector in the training phase validates the GoogLeNet architecture, along with its hyper-parameter configurations and the integrity of the training dataset. Performance of LB detector is carried out by introducing the AWGN channel model in the detection phase, where our LB detector is proven to be resilient to the AWGN environment in terms of PR, AP and MRE results.

Future work will be focused on improving performance of our LB detector, e.g. designing a particular lightweight network for the communication scenario, performing a thorough analysis of LB detectors and implementation of more complicated RF environments in the testing scenario.

\section{REFERENCES}

[1] J. Mitola and G. Q. Maguire, "Cognitive radio: making software radios more personal," IEEE personal communications, vol. 6, no. 4, pp. 13-18, 1999.

[2] B. A. Fette, Cognitive radio technology. Academic Press, 2009.

[3] G. Hattab and M. Ibnkahla, "Multiband spectrum access: Great promises for future cognitive radio networks," Proceedings of the IEEE, vol. 102, no. 3, pp. 282-306, 2014.

[4] Y. Zeng, Y.-C. Liang, A. T. Hoang, and R. Zhang, "A review on spectrum sensing for cognitive radio: challenges and solutions," EURASIP Journal on Advances in Signal Processing, vol. 2010, p. 2, 2010.

[5] T. Yucek and H. Arslan, "A survey of spectrum sensing algorithms for cognitive radio applications," IEEE communications surveys \& tutorials, vol. 11 , no. 1 , pp. 116-130, 2009.

[6] R. I. C. Chiang, G. B. Rowe, and K. W. Sowerby, "A quantitative analysis of spectral occupancy measurements for cognitive radio," in 2007 IEEE 65th Vehicular Technology Conference - VTC2007-Spring, April 2007, pp. 3016-3020.

[7] D. Sun, T. Song, B. Gu, X. Li, J. Hu, and M. Liu, "Spectrum sensing and the utilization of spectrum opportunity tradeoff in cognitive radio network," IEEE Communications Letters, vol. 20, no. 12, pp. 2442-2445, Dec 2016.

[8] N. Han, G. Zheng, S. H. Sohn, and J. M. Kim, "Cyclic autocorrelation based blind ofdm detection and identification for cognitive radio," in Wireless Communications, Networking and Mobile Computing, 2008. WiCOM'08. 4th International Conference on. IEEE, 2008, pp. 1-5.

[9] S. H. Sohn, N. Han, J. M. Kim, and J. W. Kim, "Ofdm signal sensing method based on cyclostationary detection," in Cognitive Radio Oriented Wireless Networks and Communications, 2007. CrownCom 2007. 2nd International Conference on. IEEE, 2007, pp. 63-68.

[10] G. Prema and P. Gayatri, "Blind spectrum sensing method for ofdm signal detection in cognitive radio communications," in 2014 International Conference on Communication and Network Technologies, Dec 2014, pp. $42-47$.

[11] X. Yan, F. Long, J. Wang, N. Fu, W. Ou, and B. Liu, "Signal detection of mimo-ofdm system based on auto encoder and extreme learning machine," in Neural Networks (IJCNN), 2017 International Joint Conference on. IEEE, 2017, pp. 1602-1606.

[12] S. Chaudhari, V. Koivunen, and H. V. Poor, "Autocorrelation-based decentralized sequential detection of ofdm signals in cognitive radios," IEEE Transactions on Signal Processing, vol. 57, no. 7, pp. 2690-2700, 2009.

[13] J. R. Uijlings, K. E. Van De Sande, T. Gevers, and A. W. Smeulders, "Selective search for object recognition," International journal of computer vision, vol. 104, no. 2, pp. 154-171, 2013.

[14] C. L. Zitnick and P. Dollár, "Edge boxes: Locating object proposals from edges," in European conference on computer vision. Springer, 2014, pp. 391-405.
[15] R. Vaillant, C. Monrocq, and Y. L. Cun, "Original approach for the localisation of objects in images," IEE Proceedings - Vision, Image and Signal Processing, vol. 141, no. 4, pp. 245-250, Aug 1994.

[16] D. G. Lowe, "Distinctive image features from scale-invariant keypoints," International journal of computer vision, vol. 60, no. 2, pp. 91-110, 2004.

[17] N. Dalal and B. Triggs, "Histograms of oriented gradients for human detection," in Computer Vision and Pattern Recognition, 2005. CVPR 2005. IEEE Computer Society Conference on, vol. 1. IEEE, 2005, pp. 886-893.

[18] R. Girshick, J. Donahue, T. Darrell, and J. Malik, "Rich feature hierarchies for accurate object detection and semantic segmentation," in Proceedings of the IEEE conference on computer vision and pattern recognition, 2014, pp. 580-587.

[19] A. Ali and W. Hamouda, "A novel spectrum monitoring algorithm for ofdm-based cognitive radio networks," in 2015 IEEE Global Communications Conference (GLOBECOM), Dec 2015, pp. 1-6.

[20] A. Nafkha, M. Naoues, K. Cichon, and A. Kliks, "Experimental spectrum sensing measurements using usrp software radio platform and gnuradio," in 2014 9th International Conference on Cognitive Radio Oriented Wireless Networks and Communications (CROWNCOM). IEEE, 2014, pp. 429-434.

[21] P. Aparna and M. Jayasheela, "Cyclostationary feature detection in cognitive radio using different modulation schemes," International Journal of Computer Applications, vol. 47, no. 21, 2012.

[22] W. A. Gardner, Statistical Spectral Analysis: A Nonprobabilistic Theory. Upper Saddle River, NJ, USA: Prentice-Hall, Inc., 1986.

[23] I. G. Anyim, J. Chiverton, M. Filip, and A. Tawfik, "Efficient and low complexity optimized feature spectrum sensing with receiver offsets," in 2018 IEEE Wireless Communications and Networking Conference (WCNC). IEEE, 2018, pp. 1-6.

[24] J. Antoni, "Cyclic spectral analysis in practice," $\mathrm{Me}$ chanical Systems and Signal Processing, vol. 21, no. 2, pp. $597-630,2007 . \quad$ [Online]. Available: http://www.sciencedirect.com/science/article/pii/S0888327006001816

[25] C. Szegedy, W. Liu, Y. Jia, P. Sermanet, S. Reed, D. Anguelov, D. Erhan, V. Vanhoucke, and A. Rabinovich, "Going deeper with convolutions," in Proceedings of the IEEE conference on computer vision and pattern recognition, 2015, pp. 1-9.

[26] T. Fang, "A novel computer-aided lung cancer detection method based on transfer learning from googlenet and median intensity projections," in 2018 IEEE International Conference on Computer and Communication Engineering Technology (CCET), Aug 2018, pp. 286-290.

[27] V. Ostankovich and I. Afanasyev, "Illegal buildings detection from satellite images using googlenet and cadastral map," in 2018 International Conference on Intelligent Systems (IS), Sep. 2018, pp. 616-623.

[28] A. Krizhevsky, I. Sutskever, and G. E. Hinton, "Imagenet classification with deep convolutional neural networks," in Advances in neural information processing systems, 2012, pp. 1097-1105.

[29] S. S. Keerthi, V. Sindhwani, and O. Chapelle, "An efficient method for gradient-based adaptation of hyperparameters in svm models," in Advances in neural information processing systems, 2007, pp. 673-680.

[30] J. Bergstra and Y. Bengio, "Random search for hyper-parameter optimization," Journal of Machine Learning Research, vol. 13, no. Feb, pp. 281-305, 2012.

[31] H. Müller, W. Müller, D. M. Squire, S. Marchand-Maillet, and T. Pun, "Performance evaluation in content-based image retrieval: overview and proposals," Pattern recognition letters, vol. 22, no. 5, pp. 593-601, 2001.

[32] L. Liu and M. T. Özsu, Encyclopedia of database systems. Springer New York, NY, USA:, 2009, vol. 6.

[33] J. Davis and M. Goadrich, "The relationship between precision-recall and roc curves," in Proceedings of the 23rd international conference on Machine learning. ACM, 2006, pp. 233-240.

[34] G. Huang and J. K. Tugnait, "On cyclostationarity based spectrum sensing under uncertain gaussian noise," IEEE Transactions on Signal Processing, vol. 61, no. 8, pp. 2042-2054, 2013.

[35] X. Chen and A. Gupta, "An implementation of faster renn with study for region sampling," arXiv preprint arXiv:1702.02138, 2017.

[36] V. Campos, B. Jou, and X. Giro-i Nieto, "From pixels to sentiment: Fine-tuning cnns for visual sentiment prediction," Image and Vision Computing, vol. 65, pp. 15-22, 2017. 
2020-04-30

\section{Learning based spectrum hole detection for cognitive radio communication}

Xu, Zhengjia

IEEE

Xu Z, Petrunin I, Tsourdos A, Ayub S. (2020) Learning based spectrum hole detection for cognitive radio communication. In: 2019 IEEE/AIAA 38th Digital Avionics Systems Conference (DASC), 8-12 September 2019, San Diego, https://doi.org/10.1109/DASC43569.2019.9081799

Downloaded from Cranfield Library Services E-Repository 